\title{
"Light-box" accelerated growth of poinsettias: LED-only Illumination
}

\author{
Charitha Weerasuriya ${ }^{1,2}$, Stewart Detez ${ }^{3}$, Soon Hock Ng ${ }^{1}$, Andrew Hughes ${ }^{3}$, \\ Michael Callaway ${ }^{3}$, Iain Harrison ${ }^{3}$, Tomas Katkus ${ }^{1}$, Saulius Juodkazis ${ }^{1,4}$ \\ ${ }^{1}$ Nanotechnology facility, Swinburne University of Technology, John st., Hawthorn 3122 Vic, \\ Australia \\ ${ }^{2}$ Ampcontrol Burn Brite Ltd. 1/100 New Street Ringwood Vic 3134, Australia \\ ${ }^{3}$ Horticultural and Environmental Technologies, Department of Trades and Engineering \\ Technologies, Swinburne University of Technology, 369 Stud Road Wantirna 3152 Vic, \\ Australia \\ 4 Melbourne Centre for Nanofabrication, the Victorian Node of the Australian National \\ Fabrication Facility, 151 Wellington Rd., Clayton 3168 Vic, Australia
}

\begin{abstract}
For the current commercialized agricultural industry which requires a reduced product lead time to customer and supply all year round, an artificial light emitting diode (LED)-based illumination has high potential due to high efficiency of electrical-to-light conversion. The main advantage of the deployed Red Green Blue Amber LED lighting system is colour mixing capability, which means the ability to generate all the colours in the spectrum by using three or four primary colour LEDs. The accelerated plant growth was carried out in a "light-box" which was made to generate an artificial day/night cycle and had a capability to tune the colour mixing ratio along the colour temperature curve of the chromaticity diagram. The control group of plants form the same initial batch was grown on the same shelf in a greenhouse at the same conditions with addition of artificial illumination by incandescent lamps for few hours. More dense and better quality (hence, commercial value) plants were grown in the light-box. Costs and efficiency projections of LED lamps for horticultural applications is discussed together with required capital investment. The total cost of the "light-box" including LED lamps and electronics was 850 AUD.
\end{abstract}

Keywords: LED, horticulture, efficiency, green technologies

\section{INTRODUCTION}

Light-emitting diodes (LEDs) are the most efficient lighting devices and used in many luminaire applications. Luminaires with LED chips have flexibility to control its light output as per the application requirements, including colour temperature, colour spectrum, and saturation. Combination of four primary colours (red, green, blue, amber) or RGBA per the CIE 1931 colour space chromaticity diagram can most reliably simulate the natural colours we see in nature. ${ }^{1,2}$ Due to low power consumption and efficient light controlling methods, LED chips generate less heat compared to conventional high-pressure sodium lamps used in agriculture. ${ }^{3}$ Moreover, the lifetime of LED lamps of $\sim 10$ years makes further impact for competitiveness of LED lighting applications. This introduces new engineering design capabilities in placing lamps in proximity to the plants and allows the use vertical organisation of plants on shelves.

The main objective of the current LED lighting for plant growth applications is to investigate behaviour of the plant growth under artificial lighting. One example application where a circadian rhythm for a plant should be controlled is an accelerated industrial growth of Christmas poinsettia (euphorbia pulcherrima) for blossoming

Further author information:

E-mails: C.W. weerasuriya@gmail.com; S.D. sdetez@swin.edu.au; S.J. sjuodkazis@swin.edu.au, Telephone: +61 39214 87178 


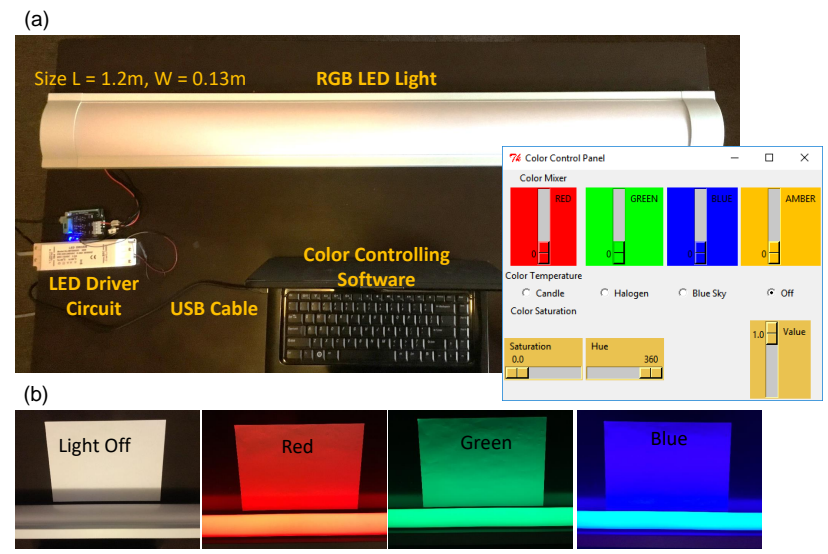

Figure 1. (a) Lamp with RGB LED-ribbons and controlling parts; separate single color-ribbons were used. Inset (rightside) shows the GUI for RGBA control of the LED-ribbons. (b) White paper sheet illuminated with different colors.

(colour change). In Australia (southern hemisphere), poinsettia shoots are planted in June for blossoming half-ayear later in December. This corresponds to an unnatural winter-to-summer cycle change for the Mexican plant, originally from the northern hemisphere.

Here we report a study of poinsettia growth using LED artificial lighting. Full control over natural lighting (intensity and colour) by using LED lamps in a fully controlled greenhouse environment was achieved and an accelerated growth cycle of poinsettias has been demonstrated. The test patch was $\sim 1 \mathrm{~m}^{2}$ and was fully separated from the outside greenhouse lights. One of the expected outcome of the project is to develop a light controlling system for accelerating plant growth. ${ }^{4,5}$ The capital cost investment into the LED lighting system and the "light-box" for plant growth tests in a greenhouse is presented to facilitate estimation of technology readiness and competitiveness.

In particular for the tested plant poinsettia, Euphorbia pulcherrima, replication of shorter days triggering the colour change in the bracts (modified leaf stems) was tested under LED artificial illumination and altered day-night cycle. ${ }^{6}$ How specific spectrum and/or intensity effects for the bracts color change is currently not known.

\section{SAMPLES AND PROCEDURES}

\subsection{Pointsettias}

Pointsettia, Euphorbia pulcherrima, is a commercially grown plant, native to Mexico, well known for its red and green foliage, used as a floral display at Christmas time. The red colour isnt the flowers but coloured bracts located around the insignificant white/cream flowers.

The plants are purchased as plugs, small plants with well-established and independent root systems, from Ball Australia in August. The plugs are then planted into larger pots for growing on for sale pre-Christmas. Plants are sold in either singles or triples in each pot. The latter producing a larger fuller floral display.

The plants are grown in a controlled environment to produce a crop at its best ready for Christmas. Poinsettias are tropical plants and in Melbourne the crop begins its production process in winter. Therefore, they are grown within a controlled environment that reproduces the plants normal growing requirements, warmth and light. What triggers the plants bracts to change from a green to the bright red colour is the shorter periods of sunlight the plant experiences with the onset of winter. When the production crop of poinsettia plants have grown to a specified size, the plants are then exposed to shorter periods of sunlight, triggering the colour change in the bracts. $^{7}$ The controlled growing space has a roof blind, that when closed, reduces the natural light the plants are exposed to, simulating shorting days and the onset of winter. The plants react, changing colour, producing the red blooms poinsettias are known for. 

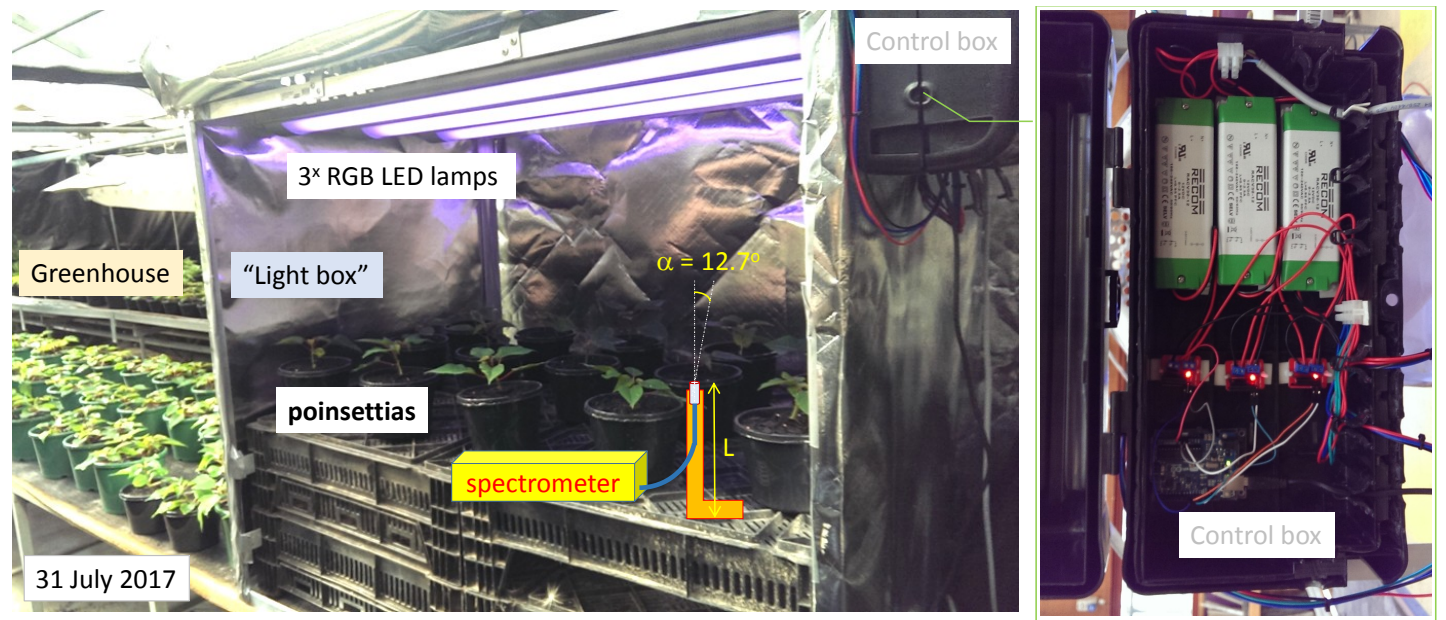

Figure 2. Start of experiment at Wantirna greenhouse using RGB LED lamp. Color spectrum was set in a "light-box": Red $(640 \mathrm{~nm}) 100 \%$, Blue $(470 \mathrm{~nm}) 100 \%$ and Green $(525 \mathrm{~nm}) 50 \%$. Spectrum and intensity of light at different heights, $L$, and at different locations inside "light-box" and greenhouse was measured with a fiber-coupled portable Ocean Optics (OCEAN-FX-VIS-NIR) spectrometer.

\subsection{DIY: infrastructure, lights, and electronics}

To design and develop a customized electronic product requires a lot of effort and funding. However, thanks to open source electronics modules, many kinds of electronic DIY projects are achievable within affordable budget. The light-box is one such DIY project attempted for this study and was designed by using open source off-the-shelf components.

The "light-box" of $\sim 1 \mathrm{~m}^{2}$ was made out of light weight aluminium and covered with a reflective Al-foil. The coating aimed to homogenise the side-illumination of plants due to reflection from three LED lamps placed at the ceiling of the "light-box". The "light-box" was $1200 \mathrm{~mm}$ (Length) $\times 800 \mathrm{~mm}$ (Width) × $900 \mathrm{~mm}$ (Height) made from Aluminium angle $40 \mathrm{~mm} \times 40 \mathrm{~mm} \times 1.4 \mathrm{~mm}$ and Aluminium flat bar $30 \mathrm{~mm} \times 3 \mathrm{~mm}$, pop rivets, reflective silver film (Ametelin, Silversark), insulation and ducting tape. An approximate cost of materials was 150 AUD and time to build 3 hours.

In the light box, the combined on-chip RGB LED strips have been used to produce all the colours in the visible spectrum. In addition, electronics included one $12 \mathrm{~V}, 2.5 \mathrm{~A}$ (up to $30 \mathrm{~W}$ power) constant voltage LED driver, Aurdino Uno module to control LED spectrum and communicate with the host, power metal oxide semiconductor field-effect transistor (MOS-FET) modules for switching and a light fixture of the standard fluorescent lamp.

The RGB LED strip contains 60 (Red), 60 (Green), 60 (Blue) chips per meter, and each individual chip is capable of generating $250 \mathrm{mcd}, 880 \mathrm{mcd}, 180 \mathrm{mcd}$ red, green and blue luminous intensities (a luminous flux per unit solid angle $1 \mathrm{~cd}=1 \mathrm{~lm} / \mathrm{srad}$ ), respectively, at nominal $20 \mathrm{~mA}$ current. Furthermore, wavelength of each colour is $640 \mathrm{~nm}$ Red, $525 \mathrm{~nm}$ Green, $470 \mathrm{~nm}$ Blue. In a single light fixture, three one-meter strips are used to increase luminous intensity.

Electronics and RGB-LEDs were bought online (Ebay). The light fixtures were adapted from fluorescent lamps (DIY Bunnings, Mercator Cannes lamp 32 AUD). Lamp diffuser was essential for color mixing by scattering (Fig. 1), however, caused some intensity loss. All electronics and power supply was placed in hermetic box and placed outside the "light-box". The cost per LED lamp composed of three LED-ribbons and driver electronics was following: RGB LED-ribbon of 60-LEDs was 15 AUD, electronic drivers were 50 AUD.

The experiment was controlled and data was collected using an in-house made program (Python coding) for color mixing, run from a portable computer (see the user interface in the inset of Fig. 1(a)). Once lighting conditions: color intensities, temporal exposure patterns were setup, the electronics ran independently.

For measurements of intensity and spectrum, a portable spectrometer Ocean Optics (OCEAN-FX-VIS-NIR) equipped with a 400- $\mu$ m-core fiber (QP400-2-VIS-NIR) of numerical aperture $N A=0.22 \pm 0.02$ was used. 
Illuminance - a luminous flux incident on a surface - was measured with a hand-held luxmeter QM1587 (Digitech Ltd.) to compare different light flux in greenhouse and light-box; $1 \mathrm{~lx}=1 \mathrm{~lm} / \mathrm{m}^{2}$. e.g. a sunshine (not direct) amounts to $10-25 \mathrm{klx}$.

\subsection{Color mixing algorithm}

As explained above, the main idea is to control RGBA colours to generate visible spectrum. However, it is difficult to find RGBA LED strips or panels in the open electronic market. Therefore, RGB strips were selected at this initial state (Until A-amber ribbons are commercially available at the same price as the RGB). With RGBA colour, there are many parameters or configuration to set. At this moment, the focus is to monitor plant growth by manipulating day/night cycles. Day/night cycle can be simulated by controlling the correlated colour temperature of the chromaticity diagram.

Color rendering was realised following four-color RGBA color mixing, ${ }^{1}$ which allows the best coverage of color space and can achieve natural lighting, tailor more settle nuances of color dullness, intensity, hue and amplitude. Software to control LED lamp was written using Python coding and had RGBA functionality (Fig. 1(a)).

In this project, color rendering is based on varying spectral power between the green and red regions of CIE 1931 xy chromaticity diagram at the wavelength from $530 \mathrm{~nm}$ to $620 \mathrm{~nm}$. The white light falls on AGB and RGB triangle. ${ }^{1}$ Hence, the white light can be generated with the mixture of spectral powers $S_{A G B}$ or $S_{R G B}$ or linear mixture of both by controlling red and amber color ratio: ${ }^{1}$

$$
S_{R G B A}=\sigma S_{A G B}+(1-\sigma) S_{R G B}
$$

where $\sigma$ is a weight parameter $0<\sigma<1$. Radiant flux is calculated by the following equations:

$$
\begin{aligned}
& R_{R G B A}=(1-\sigma) R_{R G B}, \\
& A_{R G B A}=\sigma A_{A G B}, \\
& G_{R G B A}=\sigma G_{A G B}+(1-\sigma) G_{R G B}, \\
& B_{R G B A}=\sigma B_{A G B}+(1-\sigma) B_{R G B} .
\end{aligned}
$$

The rule above was used to set individual color intensity by the electrical current.

\section{RESULTS AND DISCUSSION}

LED lamps for experiments of plant growth under artificial illumination are commercially available (e.g., Hortiled, Ledigma Ltd.), however, at a prohibitively high cost $\left(>10 \mathrm{k} \mathrm{USD} / \mathrm{m}^{2}\right)$ for field experiments in a greenhouse. In Sec. 2 we showed price estimates which compound to 850 AUD for the $\sim 1 \mathrm{~m}^{2}$ "light-box" (prices mid-2017).

\subsection{Lighting conditions}

Colour temperature can be realised using the RGB mixing. By manipulating colour temperature, it is possible to produce a wide range of white light output. For example, $1500 \mathrm{~K}$ to almost $10000 \mathrm{~K}$ depending on the LED chip quality. One of the controlling factors is to generate artificial day and night cycles to test plant response. The day and night feeling can be created by moving the colour mixing ratio along the colour temperature curve of the chromaticity diagram. For example, a colour temperature of $6000 \mathrm{~K}$ produces something similar to natural sky blue, cool white, while a colour temperature of $3000 \mathrm{~K}$ or less creates warm white. One of the main observation is to study how plant growth is affected by the day and night cycle.

For comparison of lighting conditions in the "light-box" and greenhouse, where commercial growth of poinsettias was carried out, we used a portable Ocean Optics spectrometer and a lux-meter. The optical fiber coupler has effective numerical aperture of $N A=n \sin \alpha \simeq 0.22$ and was collecting light form a half-angle of $\alpha \simeq 12.7^{\circ}$; $n=1$ is the refractive index of air. The corresponding solid angle was $\Omega=2 \pi(1-\cos \alpha) \simeq 0.15 \operatorname{srad}$.

Figure 3(a) shows spectra measured in the "light-box" and in the greenhouse. Only RGB components are present in spectrum of the LED lamp compared with the spectrally broader spectrum from natural sun light inside the greenhouse. This makes a darker appearance of lighting conditions inside "light-box" (Fig. 2). The 

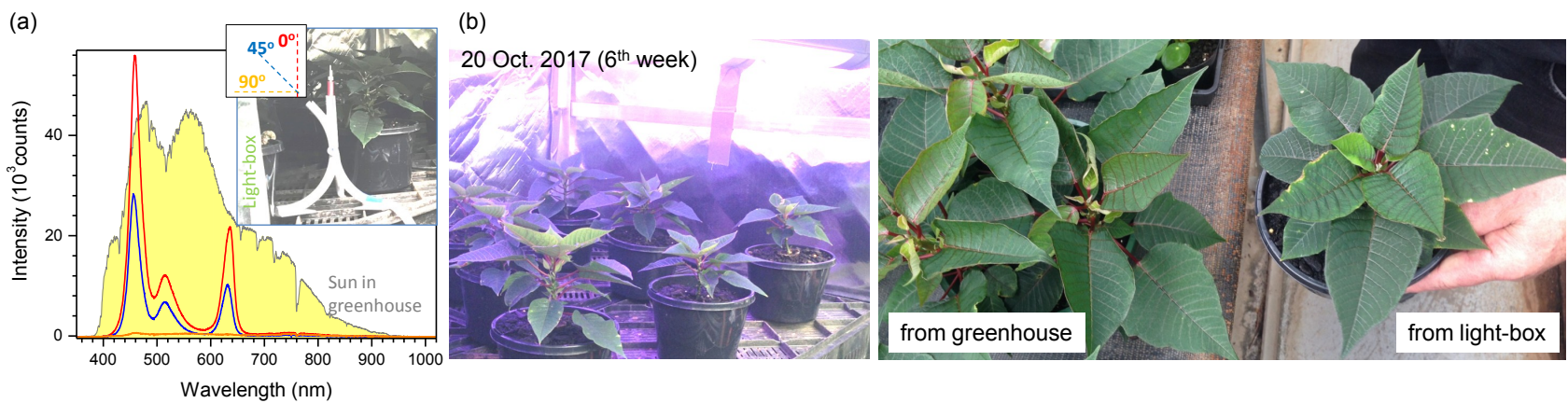

Figure 3. (a) Illuminance spectra in "light-box" at three different orientation angles of the fiber coupler shown in the inset; inset-photo shows 3D-printed stand of fiber-coupler in the measurement position. Illuminance of the Sun in the greenhouse was measured at $0^{\circ}$ (up-ward orientation) on 27 Oct. 2017 around 2:00 pm; all spectra taken with integration time of $20 \mathrm{~ms}$. Integrated area difference between Sun and LED spectra is 24.5\%; luminance ratio for the LED lamp $I_{45^{\circ}} / I_{0^{\circ}}=47.2 \%$. The (b) Poinsettias on 6 th week of experiment.

difference was quantified by calculating the area below the luminance curves (in Fig. 3(a)). Only $\sim 25 \%$ of luminance was inside the light-box. Considering natural light cycle in the greenhouse and 24/7 illumination in the light-box, the difference in light dose reaching plant was more than twice. However, the rate of biomass production for plants under natural greenhouse illumination was only slightly faster as compared with the light-box where the dose of light received by plants was less than half.

Uniformity of intensity and spectrum over the floor of the "light-box" was measured with separation of $\sim 30 \mathrm{~cm}$ (typical distance between plants) at the level of top leaves $L \simeq 30 \mathrm{~cm}$ (Fig. 2). Since the LED lamps with diffusers were used, the uniformity of illumination of the growth area approximately $70 \mathrm{~cm}$ from the lamps was uniform within $\sim 15 \%$ difference. Interestingly, luxmeter measurements are less informative since it has sensitivity maximised at the green part of the spectrum $500-530 \mathrm{~nm}$ where there was much less light emitted from the LED lamp. Luxmeter reading inside the light box was only $1.6 \%$ of that in the greenhouse.

Hardware control and monitoring was realised from a personal computer, however, a remote control and data collection can be realised as we have demonstrated in another application of RGBA-lighting for experiments with

(a)

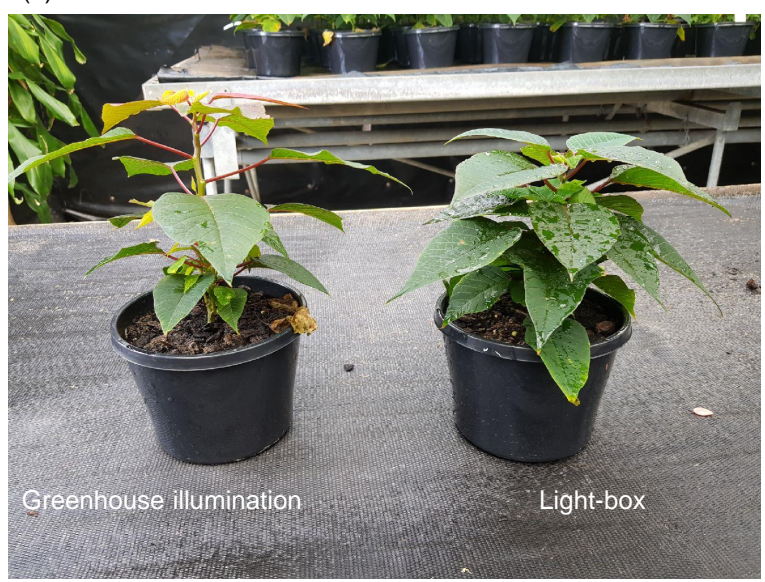

(b)

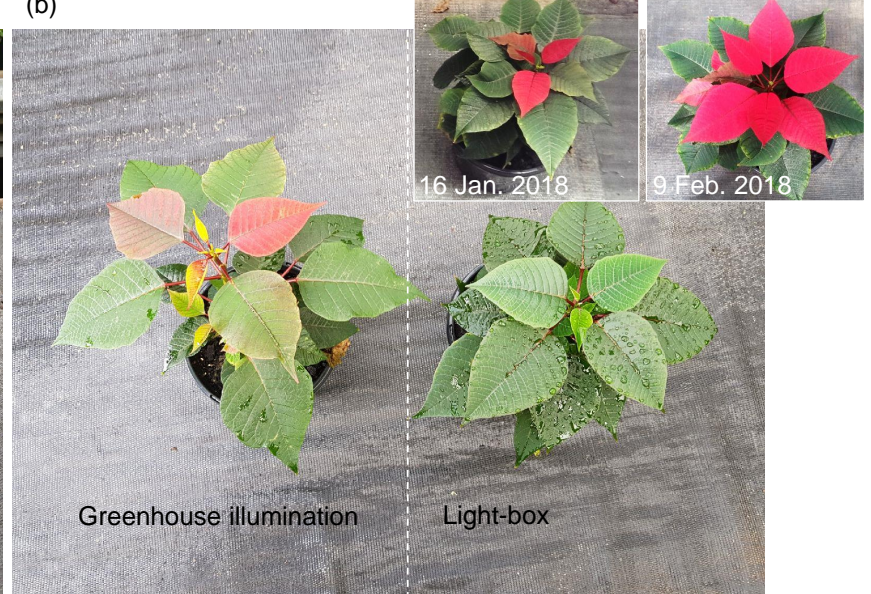

Figure 4. December 6, 2017. (a) Side-view photos reveal that plants were taller with natural greenhouse illumination (which was initially lengthened with halogen lamps for an extra 4 hours and then was switched off for the final stage of leaf coloration). (b) The top-view images for the same plants as in (a). The plant in the light-box had a light totally switched off for the night time, which was applied a two weeks later (on Dec. 6; the day pictures were taken) as compared to the start of a shorter day time illumination in the main greenhouse. The insets in (b) show the plant from the light-box on 16 January and 9 February 2018 . 
psychological effects related to color. ${ }^{8}$

\subsection{Field test: July-December}

After 6 weeks of growth (Fig. 3(b)), poinsettias from the "light-box" where slightly smaller. This correlates with an overall light intensity which was smaller for the "light-box". The greenhouse had illumination by additional eight incandescent $20 \mathrm{~W}$ lights for the additional 4 hours per day. Hence, photosynthesis is producing smaller bio-mass. Another contributing factor might be a closer proximity to the light source in the "light-box" which reduces competition for light more expressed in the greenhouse. Figure 4 illustrates this with a side-view image taken when plants entered the final stage of the growth cycle and additional illumination was stopped in the greenhouse on the 21st of November. For the "light-box", the move from 24/7 illumination to a normal day/night cycle occurred on the 6th of December. A more dense and a better structured plant was formed in the light-box. A side-view also reveals a denser growth while the top-view shows a better structural form for the light-box poinsettia. As a commercial product, the plant grown in the light-box is of a greater value.

Due to comparatively small enclosure of plants in the "light-box", plants were slightly more affected by parasites, however, this is a natural consequence of a lower efficiency of ventilation.

\section{CONCLUSIONS AND OUTLOOK}

We have demonstrated a simple do-it-yourself approach in realisation of LED-lamps for agricultural applications. A full cycle of commercial growth of poinsettias during July-December was carried out in the "light-box" conditions. Noteworthy, the illumination cycle transition occurred 3 weeks later for the "light-box" plants; after this transition, the onset of colour change took 1 week longer than their greenhouse counterparts. Luminance in the light-box was less than half that of the greenhouse, however, bio-mass production differed by less than $20 \%$. RGB LED lamp was illuminating plants all the time without day-night cycle for the first 10 weeks.

This work shows that field test using affordable infrastructure is in reach and scientific questions on optimal spectral conditions, circadian cycle control, etc., can be now tested for different decorative and edible plants. The "light-box" could be combined with hydroponics for growth of vegetables in demanding conditions such as encountered in Antarctic research stations where hydroponics are used.

High efficiency and longevity of LEDs with a trend of decreasing price should make this technology commercially viable. By combining artificial LED lighting and quantitative monitoring of photosynthesis with PhotosynQ hand-held technology, new protocols of efficient growth of plants will be developed. It has been already demonstrated that additional illumination by $638 \mathrm{~nm}$ wavelength light three days before harvesting of lettuce, marjoram, and green onions resulted in a 44-to- $65 \%$ reduction of nitrates and increase of nutritionally valuable carbohydrates. ${ }^{9}$

\section{ACKNOWLEDGMENTS}

This work was initiated as part of the technology readiness VG15038 grant: Investigating novel glass technologies and photovoltaics in protected cropping (the work package: LED illumination for the indoor plant growth). This project was supported by Nanotechnology facility of Swinburne University via industrial focus initiative of 2017.

\section{REFERENCES}

[1] Žukauskas, A., Vaičekauskas, R., Vitta, P., Tuzikas, A., Petrulis, A., and Shur, M., "Color rendition engine," Optics Express 20(5), 5356 - 5367 (2012).

[2] Ledigma Ltd., "Color rendition engine: http://demo.lrg.projektas.vu.lt/lcq/en/simulation/engine," (visited on 22 Oct. 2017).

[3] Morrow, R., "Led lighting in horticulture," Hortscience 43, 1947-1950 (December 2008).

[4] Choi, H. G., Moon, B. Y., and Kang, N. J., "Effects of led light on the production of strawberry during cultivation in a plastic greenhouse and in a growth chamber," Scientia Horticulturae 189(Supplement C), 22 - 31 (2015).

[5] Wojciechowska, R., Dugosz-Grochowska, O., Koton, A., and upnik, M., "Effects of led supplemental lighting on yield and some quality parameters of lamb's lettuce grown in two winter cycles," Scientia Horticulturae 187(Supplement C), $80-86$ (2015). 
[6] Islam, M. A., Kuwar, G., Clarke, J. L., Blystad, D.-R., Gislerd, H. R., Olsen, J. E., and Torre, S., "Artificial light from light emitting diodes (leds) with a high portion of blue light results in shorter poinsettias compared to high pressure sodium (hps) lamps," Scientia Horticulturae 147(Supplement C), 136 - 143 (2012).

[7] Embry, J. L. and Nothnagel, E. A., "Leaf senescence of postproduction poinsettias in low-light stress," Journal of the American Society for Horticultural Science 119(5), 1006-1013 (1994).

[8] Weerasuriya, C., Remote color control of a LED-lamp for human judgement about color (EEE80017 project), Master's thesis, Nanotechnology facility, Swinburne University of Technology (2016).

[9] Samuolienè, G., Urbonavičiūtè, A., Duchovskis, P., Bliznikas, Z., Vitta, P., and Žukauskas, A., "Decrease in nitrate concentration in leafy vegetables under a solid-state illuminator," Hortiscience 44(7), 1857-1860 (2009). 